\title{
Bioanalysis
}

\section{Cheers: cracking open the bottleneck of extraction in bioanalysis}

\author{
"There is no one-size-fits-all solution and this means there are many competing \\ interests that make this a complicated area."
}

Keywords: extraction $\bullet$ MS $\bullet$ point-of-care testing $\bullet$ sampling

While nothing is certain in life - except perhaps death and taxes - two areas have emerged in the field of bioanalysis that are certain: MS and point-of-collection testing (POCT). The value of these is beyond dispute [1-4]. MS, alone, or hyphenated with chromatography (or electrophoresis), provides unprecedented selectivity with near unambiguous identification ability of just about everything you could ever want to analyze. POCT aims to bring us into the knowledge-immediacy culture where we want to know the answer to everything now. With immediate knowledge of everything, we can ultimately make the best choice at that point to inform future action, for example, to undertake more rigorous medical tests or to begin or adjust treatment.

\section{The common challenge}

What both of these certainties have in common is the challenge in the analytical process of isolating and detecting the target(s) from a complex mixture of molecules. It is worth thinking about this for a second to truly understand the nature of the challenge. Measuring pharmaceutical concentrations in blood for therapeutic drug monitoring requires detection of a target drug and perhaps its metabolites $(\mathrm{pg}-\mu \mathrm{g} / \mathrm{ml})$ from the cells ( 5 billion $/ \mathrm{ml}$ ), proteins $(80 \mathrm{mg} / \mathrm{ml}$ ) and salt $(10 \mathrm{mg} / \mathrm{ml})$. Proteomics and metabolomics requires not just the determination of a few things, but tens of thousands of proteins or metabolites, that can differ in concentrations by over six orders of magnitude! And finally, the diagnostic potential of circulating tumor cells (CTCs) is emerging as one of the battlegrounds to inform the treatment of cancer, but ideally this requires the detection of one CTC in your blood stream, although current diagnostic approaches target one CTC/ $\mathrm{ml}$. There is no one-size-fits-all solution and this means there are many competing interests that make this a complicated area.

\section{Selectivity: the key to simplicity}

From a fundamental perspective, all of the examples above require 'extraction' of the target to facilitate detection, but differ in the ways in which that can be done. One of the simplest and oldest approaches is to be specific and use antibodies, aptamers and/or enzymes and their exquisite selectivity to 'extract' the target from the matrix. But this is just the tip of the iceberg - they make brilliant integrated sensors for POCT because they can perform all functions in the analytical process: matrix isolation, concentration and providing the analytical response. Using a combination of antibodies and enzymes can produce amazing sensitivity, with the ability to detect a single molecule [5]. This is an amazing outcome and you cannot get much lower than single molecule detection, but the next challenge is just as hard - doing it in a large volume. CTC tests, for example, use several milliliters of sample because this is the volume required to provide a sufficiently large enough volume to be able to make an accurate measurement, not because of a lack in ability to detect single cells. Will it ever be possible to diagnose one CTC in your blood stream?

\section{When you cannot be selective?}

If there is not anything selective for your target, then the fallback is to extract the mole-

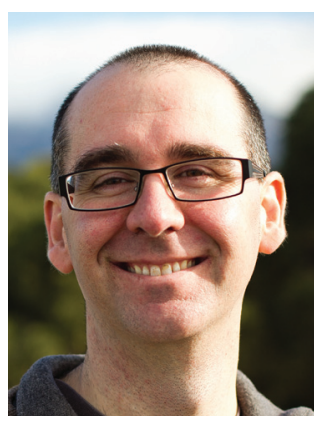

Michael C Breadmore Australian Centre for Research on Separation Science (ACROSS), School of Chemistry, University of Tasmania, Private Bag 75, Hobart, Tasmania 7001, Australia

and

ARC Training Centre for Portable Analytical Separation Technologies (ASTech), School of Chemistry, University of Tasmania, Private Bag 75, Hobart, Tasmania 7001, Australia

Tel.: +61362 262154

Fax: +61 362262858

mcb@utas.edu.au 
cules onto a solid phase (solid-phase extraction) or into an immiscible liquid phase (liquid-phase extraction) and these are the workhorses of the modern bioanalytical laboratory. Through appropriate phase and solvent selections, it is possible to isolate and concentrate a collection of molecules that have similar chemical properties from the matrix, and provide them in a form suitable for analysis. These are powerful approaches to sample treatment, but the laborious nature and large volumes required make them unattractive. One can be solved through robotic instrumentation, while the other can be solved through miniaturization, and together this provides a solution to the problem, but arguably what is needed is something simpler. An elegant case in point is the advent of solid-phase microextraction over 2 decades ago [5-7]. The key innovation was to place a small fiber of solid-phase directly into the sample and then to thermally desorb the targets from the fiber in the GC instrument. While not without its drawbacks, this is about as simple as it gets and was a significant advance in the simplification of sample treatment. It is the drive toward simplification that needs to occur, particularly if POCT devices that cover more fully the entire laboratory range of techniques are to be developed. This is one of the challenges inhibiting the development of sophisticated lab-on-a-chip devices as we move beyond the ground-breaking Medimate ${ }^{\mathrm{TM}}$ electrophoretic analyzer for the mood stabilizer lithium $[8,9]$ and toward more generic and universal approaches for a broader range of small molecule pharmaceuticals [10,11].

\section{"...extract the molecules onto a solid phase} (solid-phase extraction) or into an immiscible liquid phase (liquid-phase extraction) and these are the workhorses of the modern bioanalytical laboratory."

\section{Equally as effective: removing the problem}

The two examples above focus on removing the target from the matrix by one means or another, but an equally useful strategy is to do the reverse and to remove the matrix from the target. This is the concept behind a simple protein precipitation, although the downside to this particular example is a slight dilution which can compromise the sensitivity. This strategy has also been used widely in proteomics, particularly to remove the most abundant proteins in the sample (album, Ig) and extend the coverage of the analysis to lower abundant

\section{References}

1 Gomez FA. The future of microfluidic point-of-care diagnostic devices. Bioanalysis 5(1), 1-3 (2013). proteins. But does this concept has broader applicability? What else can be removed from the sample to facilitate a more useful and versatile extraction?

\section{Is doing nothing an option?}

Ultimately the simplest approach is one in which no treatment is required at all - and yes this is possible. Ambient MS has pioneered the direct analysis of residue on surfaces, and has even shown to be useful for the detection of ingested pharmaceuticals secreted through human skin $[12,13]$. This represents an exciting development in the field of bioanalysis, but not everything can penetrate skin. So, the question arises as to where else this may lead? Is it possible to analyze the smallest, tiniest fraction of a drop of blood on the skin instead? The continued evolution of MS will see small, portable instruments become widely available in the next decade or two. Just what sample treatment strategies will be needed to fully realize the potential?

\section{Looking to the future}

While I do not know what the future holds, I am certain that there will be some exciting developments in this field over the next decade. The last decade has seen an incredible number of new approaches that are quicker, simpler and more powerful than anything that has ever come before, and this is set to continue as new materials with distinct chemical and physical properties are being developed. Combined with further instrumental developments, to improve the sensitivity and selectivity of detection, it will change the requirements of the sample treatment process that will ultimately see a move toward the simplest approaches being used. Equally as important is the translation of these key developments into commercially viable products that will have uptake within the bioanalytical community.

Financial \& competing interests disclosure

The author acknowledges the Australian Research Council for funding through the Future Fellowship scheme (FT130100101) and the Industrial Transformation Training Centre scheme (IC140100022), through which he is collaborating with Trajan Scientific and Medical. The author has no other relevant affiliations or financial involvement with any organization or entity with a financial interest in or financial conflict with the subject matter or materials discussed in the manuscript apart from those disclosed.

No writing assistance was utilized in the production of this manuscript.

2 Osiri JK, Shadpour H. Toward point-of-care microchip profiling of proteins. Bioanalysis 2(10), 1745-1754 (2010).

3 Bhushan R, Dubey R. Integrated lab-on-chip and mass spectrometry: recent advances in bioanalysis. Bioanalysis 6(14), 1875-1877 (2014). 
eave $M$. The strengths of mass spectrometry are not just sensitivity and selectivity. Bioanalysis 3(3), 245-247 (2011).

5 Rissin DM, Kan CW, Campbell TG et al. Single-molecule enzyme-linked immunosorbent assay detects serum proteins at subfemtomolar concentrations. Nat. Biotechnol. 28(6), 595-599 (2010).

6 Louch D, Motlagh S, Pawliszyn J. Dynamics of organic compound extraction from water using liquid-coated fused silica fibers. Anal. Chem. 64(10), 1187-1199 (1992).

7 Pawliszyn JB, Alexandrou N. Indirect supercritical fluid extraction of organics from water matrix samples. Water Pollut. Res. J. Can. 24(2), 207-214 (1989).

8 Eijkel J. Chip-based capillary electrophoresis platforms: toward point-of-care applications. Bioanalysis $7(11)$, 1385-1387 (2015).
9 Floris A, Staal S, Lenk S et al. A prefilled, ready-to-use electrophoresis based lab-on-a-chip device for monitoring lithium in blood. Lab Chip 10(14), 1799-1806 (2010).

10 Shallan AI, Gaudry AJ, Guijt RM, Breadmore MC. Tuneable nanochannel formation for sample-in/answer-out devices. Chem. Commun. (Camb). 49(27), 2816-2818 (2013).

11 Shallan AI, Guijt RM, Breadmore MC. Electrokinetic size and mobility traps for on-site therapeutic drug monitoring. Angew. Chem. Int. Ed. Engl. 54(25), 7359-7362 (2015).

12 Katona M, Dénes J, Skoumal R, Tóth M, Takáts Z. Intact skin analysis by desorption electrospray ionization mass spectrometry. Analyst 136(4), 835-840 (2011).

13 Takáts Z, Wiseman JM, Gologan B, Cooks RG. Mass spectrometry sampling under ambient conditions with desorption electrospray ionization. Science 306(5695), 471-473 (2004). 